Design Methodologies and the Limits of the Engineering-Dominated Conception of Synthetic Biology

\author{
ljäs, Tero Tapio
}

2019-03

ljäs , T T 2019 , ' Design Methodologies and the Limits of the Engineering-Dominated

Conception of Synthetic Biology ' , Acta Biotheoretica , vol. 67 , no. 1, pp. 1-18 . https://doi.org/10.1007/s10441-018

http://hdl.handle.net/10138/305993

https://doi.org/10.1007/s10441-018-9338-7

unspecified

acceptedVersion

Downloaded from Helda, University of Helsinki institutional repository.

This is an electronic reprint of the original article.

This reprint may differ from the original in pagination and typographic detail.

Please cite the original version. 


\title{
Design methodologies and the limits of the engineering-dominated conception of synthetic biology
}

\section{Tero Ijäs}

tero.ijas@helsinki.fi

University of Helsinki, Department of Political and Economic Studies, PO Box 24 (Unioninkatu 40), 00014 University of Helsinki, Finland.

\begin{abstract}
Synthetic biology is described as a new field of biotechnology that models itself on engineering sciences. However, this view of synthetic biology as an engineering field has received criticism, and both biologists and philosophers have argued for a more nuanced and heterogeneous understanding of the field. This paper elaborates the heterogeneity of synthetic biology by clarifying the role of design and the variability of design methodologies in synthetic biology. I focus on two prominent design methodologies: rational design and directed evolution. Rational design resembles the design methodology of traditional engineering sciences. However, it is often replaced and complemented by the more biologically-inspired method of directed evolution, which models itself on natural evolution. These two approaches take philosophically different stances to the design of biological systems. Rational design aims to make biological systems more machine-like, whereas directed evolution utilizes variation and emergent features of living systems. I provide an analysis of the methodological basis of these design approaches, and highlight important methodological differences between them. By analyzing the respective benefits and limitations of these approaches, I argue against the engineering-dominated conception of synthetic biology and its "methodological monism," where the rational design approach is taken as the default design methodology. Alternative design methodologies, like directed evolution, should be considered as complementary, not competitive, to rational design.
\end{abstract}

Keywords: Synthetic biology; Engineering; Design; Rational Design; Directed Evolution

\section{Introduction}

Synthetic biology is framed as a project that aims to make biotechnology more like engineering.

According to this approach, biological systems should be treated and constructed using the same principles as artificial devices in the engineering sciences (Cambray, Mutalik, and Arkin 2011). The rise of synthetic biology is partially explained by the recent development of biotechnology, which has led to the discovery and characterization of numerous molecular parts. The availability and utilization of these parts has become a central methodological principle in the engineering- 
dominated account of synthetic biology and in its design approach. An example of this engineering ethos can be seen, for example, in the Strategic Vision of the European Research Network of Synthetic Biology, which considers synthetic biology a "way of looking at living systems, not as complex objects to be thoroughly understood, but as sources of amazing building blocks that can be retrieved from their natural context, reshaped, standardized to fit a given specification and used for a purpose different from their original raison d'être." (ERASynBio 2014, 2).

The application of these engineering principles to biological systems has led synthetic biologists to emphasize the importance of design work and to systematize their design methodology. The most prominent of these design approaches is rational design, a component-focused approach that resembles the design methodology of traditional engineering sciences, such as mechanical and electrical engineering (Giese et al. 2013; Heinemann and Panke 2006). Rational design aligns with the engineering-dominated account of synthetic biology, whose proponents argue that biologists should "start thinking of designing biological parts in a forward engineering manner" (Heinemann and Panke 2009, 392). Consequently, they consider the novelty of synthetic biology to be based on the application of rational design, and argue that in the future rational design should be the primary design methodology of synthetic biology (Agapakis 2014; Bujara and Panke 2010).

Subsequently, most philosophical literature approaches synthetic biology as an engineering discipline and rational design as its central methodology. This simplification allows one to draw an analogy between biological and man-made systems, and to bring the philosophical discussion concerning technical artifacts to the domain of synthetic biology. Following this analogy, philosophers have discussed, for example, its connections to mechanistic philosophy (Holm 2015) or the application of machine metaphors to biology (Boudry and Pigliucci 2013). As philosophically practical as this simplification is, it unfortunately gives a narrow view of synthetic biology. Despite this vision and similar engineering-inspired definitions, synthetic biology is a more heterogeneous discipline. Like many emerging fields in biology, synthetic biology encompasses 
several different methods and researchers from multiple disciplines, including both basic and applied research (Knuuttila and Loettgers 2013a), and its methods have been applied from gene circuits to protocell creation, and from DNA editing to whole-genome engineering (O'Malley et al. 2007).

I aim to elaborate the heterogeneity of synthetic biology by examining the limits of rational design and the principles on how design approaches are applied. Though often a useful and powerful methodology, rational design fails in some cases due to the complexity and context-sensitivity of living systems, and thus alternative design methodologies are widely used in synthetic biology. One of these alternative design approaches is directed evolution, which utilizes more biologicallyinspired design principles. Directed evolution models itself on natural and artificial evolution, and aims to improve and modify systems by guiding them to evolve in a certain direction. Directed evolution and similar evolutionary approaches have received less attention in philosophical literature than rational design methods. Furthermore, directed evolution is sometimes represented (e.g., (Bujara and Panke 2010; O’Malley 2011)), not as an independent design approach, but as another form of tinkering and kludging that allows synthetic biologists to produce desired functionality when rational design fails.

In Sections 2 and 3, I present a systematic analysis of the requirements, benefits and limitations of both rational design and directed evolution design approaches. This paper has three objectives. First, by giving a systematic review of two different design approaches, I offer a more nuanced view of the current design methodologies of synthetic biology. This subsequent comparison of rational design and directed evolution design approaches is given in Table 1. It provides a summary of these design approaches and their methodological bases. It also identifies the important methodological differences between them, which I will detail in the later sections of this paper. Second, I argue against the framing of the role of directed evolution merely as supplementing rational design and allowing it to overcome some of its current technical limitations. In reality, 
directed evolution is a systematic design approach that is both common in the actual practice of synthetic biology (e.g., (Haseltine and Arnold 2007; Packer and Liu 2015; Romero and Arnold 2009)) and, as I show in this paper, methodologically distinct from rational design. And finally, by analyzing the respective benefits and limitations of these approaches, I argue against the engineering-dominated conception of synthetic biology and its "methodological monism," where the rational design approach is taken as the default design methodology. Alternative design methodologies, like directed evolution, should be considered to be complementary, not competitive or merely supplementary, to rational design. 


\begin{tabular}{|c|c|c|}
\hline & Rational Design & Directed Evolution \\
\hline Design principles & $\begin{array}{l}\text { Hierarchical bottom-up system } \\
\text { construction from standardized parts } \\
\text { to more complex devices and systems. } \\
\text { Reductionistic heuristics. }\end{array}$ & $\begin{array}{l}\text { Deliberate mutations are induced to target } \\
\text { (library generation). Mutants with desired } \\
\text { phenotypes are selected for further iterations. }\end{array}$ \\
\hline "Role model" & $\begin{array}{l}\text { Models itself after mature engineering } \\
\text { sciences and their design } \\
\text { methodology. }\end{array}$ & $\begin{array}{l}\text { Models itself after natural evolution and } \\
\text { selective breeding. }\end{array}$ \\
\hline Design tasks & $\begin{array}{l}\text { Decoupling. Standardization. } \\
\text { Hierarchy ("parts make devices and } \\
\text { devices make systems"). }\end{array}$ & $\begin{array}{l}\text { Choice of mutational target and selective } \\
\text { pressures. Definition of selection/screening } \\
\text { criteria. }\end{array}$ \\
\hline Requirements & $\begin{array}{l}\text { Prior structural knowledge. } \\
\text { Availability of well-characterized } \\
\text { (standardized) parts and modules. } \\
\text { Computational modeling tools to } \\
\text { guide device construction. }\end{array}$ & $\begin{array}{l}\text { Ability to select and screen desired phenotypes. } \\
\text { Incremental pathway of beneficial mutations to } \\
\text { desired behavior. }\end{array}$ \\
\hline Benefits & $\begin{array}{l}\text { Relative simplicity and tractability of } \\
\text { design tasks and methods. } \\
\text { Theoretically easy to design complex } \\
\text { and novel functions. Potentially very } \\
\text { powerful design approach. }\end{array}$ & $\begin{array}{l}\text { Not limited to a certain level of hierarchy. } \\
\text { Allows system improvement in the absence of } \\
\text { mechanistic detail. Environmental tuning of } \\
\text { non-functional systems. }\end{array}$ \\
\hline Limitations & $\begin{array}{l}\text { Sufficient structural knowledge and } \\
\text { mechanistic detail not available. } \\
\text { Context-sensitivity. Change in } \\
\text { functionality due to evolution. }\end{array}$ & $\begin{array}{l}\text { Decrease in mechanistic understanding and } \\
\text { tractability. Combinatorial complexity. Difficulty } \\
\text { to screen for independent properties. Increase } \\
\text { of non-modularity. }\end{array}$ \\
\hline Living systems & $\begin{array}{l}\text { Mechanization as a heuristic goal. } \\
\text { Simplification of systems and } \\
\text { suppression of variation. }\end{array}$ & $\begin{array}{l}\text { Utilization of variation, self-organization and } \\
\text { "emergent" properties of biological systems. }\end{array}$ \\
\hline $\begin{array}{l}\text { Approach to natural } \\
\text { evolution }\end{array}$ & $\begin{array}{l}\text { Seen as destructive: loss of systems } \\
\text { functionality due to evolutionary } \\
\text { change. }\end{array}$ & $\begin{array}{l}\text { Seen as (potentially) constructive: system } \\
\text { improvement through variation and selection. }\end{array}$ \\
\hline
\end{tabular}

Table 1. Comparison of rational design and directed evolution design approaches.

\section{Rational design}

Synthetic biology is often defined through its novel approach of modeling itself on the engineering sciences. Subsequently, synthetic biology has borrowed methodological principles and terms from fields like mechanical, electric and software engineering. These fields are taken to exemplify the methods of "traditional" or "classical" engineering (Heinemann and Panke 2009; Kroes 2012). One such idea of classical engineering, which synthetic biologists aim to adapt, is the higher focus on 
design and on distinguishing design process as an autonomous task, separate from the fabrication (cf. (Houkes and Vermaas 2010; Kroes 2012)). In previous biotechnology and molecular biology, design work was tied to wet lab work with no clear division between design and fabrication. However, the attempts to make biotechnology more similar to engineering have led synthetic biologists to distinguish design as an autonomous phase (Cambray, Mutalik, and Arkin 2011; Heinemann and Panke 2006). In synthetic biology, the development of design tools such as the use of computer-assisted design and the standardization of components should allow design to be separated as both a methodologically and cognitively distinct task, and allow a higher division of labor between design and wet lab experimental work (Roosth 2017).

Similar to engineering, synthetic biologists have proceeded to systematize their design methodology and develop distinct approaches to achieve reliable bioengineering of organisms with desirable traits. A systematic design approach should define the methods, heuristics and performance criteria that the engineer uses to solve design problems and to assess the success of the design (Kroes 2012). Subsequently, rational design is seen to follow the engineering ideals of synthetic biology by emphasizing the decomposability of its systems and their hierarchical and modular nature. Its prevalence has led some writers (e.g., Lewens 2013) to define textbook synthetic biology through its method of applying rational design methods to living systems.

Rational design can be described as the idea that with sufficiently well-developed methods, tools and systems, synthetic biologists should be able model and predict how modifications of the system should affect its behavior (Cambray, Mutalik, and Arkin 2011). In practice, rational design is a parts-based approach that constructs novel systems bottom-up using well-characterized parts. This means that ideally, synthetic biologists should be able to manipulate the system by manipulating its parts. Its three major principles are (1) decoupling of design tasks, (2) the use of standardized modular parts, and (3) hierarchical organization (Endy 2005; Heinemann and Panke 2006). 
Decoupling of design tasks relies on the idea that the main design goals can be divided into simpler sub-problems that are easier to tackle. Ideally, each of these design tasks could be solved semiindependently in relation to other tasks. Rational design proceeds to achieve this localized and modular problem-solving through the standardization of components. Standardization is one of the main methodological principles of the engineering-dominated synthetic biology, as it aims to characterize and define a set of standardized biological components that can be assembled to more complex systems with predictable outcomes (Endy 2005). Successful standardization makes engineering more predictable and effective, as standardized parts are expected to behave in a similar manner, and make the subsequent modifying and maintenance of the system easier. Rational design expands this ideal to biotechnology by defining standards for biomolecules. Possible components can be, for example, DNA promoter regions and regulatory, structural and reporter genes. The language of a standardized toolbox of parts reveals the engineering aspirations of synthetic biology, as synthetic biologists talk, for example, of genetic switches and circuits (Purnick and Weiss 2009). Ideally, with a sufficiently developed toolkit of standardized components, synthetic biologists should be able to solve a range of more complex biological problems by dividing up the problems and then solving the sub-problems by using appropriate parts and devices. Standardized components could be put together to form more complex modules, such as oscillators, switches or genetic circuits (Andrianantoandro et al. 2006). In turn, these second-level modules should exhibit hierarchical organization and the ability to be combined with more complex systems, such as full metabolic or signaling networks.

In properly hierarchical and modular systems, parts make devices, and devices make whole systems. In these circumstances, the main task in rational design is to carefully specify, choose and combine the individual components of the system resulting in predictable composite behavior (Cambray, Mutalik, and Arkin 2011; Haseltine and Arnold 2007). However, this requires many 
major features from parts and their interactions, and satisfying these requirements is not simple, as I will show in the next section.

\subsection{The requirements and benefits of rational design}

We have established that rational design is a parts-based approach that constructs its systems from the bottom-up by utilizing well-characterized standardized components. The success of rational design depends on the availability and performance of its components. Therefore, the question "What is required for successful rational design?" can be reformulated as "What is required from the parts in successful rational design?" In principle, the components in proper rational design should produce the sub-functions that are required to satisfy the defined design tasks. In practice, this requires several important features from components, such as modularity, predictability and stability (Cambray, Mutalik, and Arkin 2011).

The most important of these features is modularity. For successful rational design, synthetic biological system should be composed of relatively independent modules that have definable functionalities and interfaces in relation to other modules (Haseltine and Arnold 2007). Modularity is an important feature to reduce complexity and increase system robustness. It makes a system more easily decomposable and recomposable. In modular systems, parts can be manipulated, interchanged and reassembled without causing unpredictable changes to system behavior, whereas in non-modular systems, substitution, rearrangement and manipulation of some parts can affect the functionality of others (Wimsatt 2007). Non-modular systems are harder to control and predict, as local changes to one part tend to propagate through the system (Oftedal and Parkkinen 2013). One should be careful not to think of modularity as a simple dichotomy where the system is either modular or non-modular. In most systems, modularity comes in degrees and system modularity can be studied from more than one perspective (Wagner 2005). For example, the system might exhibit 
functional modularity without structural modularity in a case where there are discernible relatively autonomous sub-functions, but there are no spatial separation or physical barriers between them.

This is why the development of standardized parts is one of the main aims of rational design. For synthetic biology, standardization is based on the idea that a limited toolbox of well-characterized components could allow a synthetic biologist to design and construct a great range of functionalities in a number of domains, rather than trying to solve each problem individually. For each part, this would require the practical ability to construct a part that can be isolated and combined, and a characterization of the part's behavior, a definition of the range of its domains of application, and protocols for connecting parts (Arkin and Fletcher 2006). To achieve the sharing and circulation of standardized components among the synthetic biology scientific community, this also requires the creation of databases for the standardized parts, such as the prominent open-source BioBrick Registry, and organization of database management, such as protocols for the sharing and curation of data.

Standardized and widely available synthetic components make the design of systems relatively simpler than previously. In principle, it is easy to understand how rational design can be used to obtain novel higher-level functions in hierarchically structured systems; the more complex functionalities are the composite of the lower-level functions and are achieved by combining parts to sub-modules and sub-modules to higher systems (Haseltine and Arnold 2007). When its requirements are met, rational design offers a potentially powerful method for the design and manipulation of biological systems. Furthermore, rational design provides a good template to understand how the development of synthetic biology proceeds, namely through the characterization of more efficient, reliable and powerful components that allow the construction of a higher range of systems. The assumed plug and play functionality of rational design should make biotechnology more efficient and cost-effective, as synthetic biology laboratories or biotechnology can bypass earlier steps of development by ordering the required components from suppliers. 
Some of these benefits, like simplicity and tractability, arise from the heuristics of the traditional engineering design paradigm. Philosophers of science have discussed the importance of the "mechanistic" (Bechtel and Richardson 1993) or "reductionistic" (Wimsatt 2007) heuristics used to study complex biological systems. Bechtel and Richardson (1993) argue that scientific reasoning about complex systems proceeds through the process of decomposition and localization. This means that scientists try to explain the macro-level behavior of the system by first providing a structural and functional decomposition of the system, and then by localizing the sub-functions to the system's component parts. This is very similar to the previously discussed principles of rational design, where synthetic biologists attempt to decouple the design tasks to sub-tasks that can be solved by semi-independently using standardized components. Reductionistic heuristics work best with systems that are nearly decomposable (see (Simon 1996)), that is, when the system behavior is a composite of the functionalities of its parts. In turn, a system is considered to be integrative if its behavior depends more on the interactions between its parts than the parts' intrinsic causal powers. Integrative systems exhibit more emergent system-level behavior and are less suited to reductionistic heuristics.

I argue that the success of rational design depends on whether the designed systems follow the assumptions that reductionistic heuristics make about their objects of study. The heuristics of decomposition and localization have influenced later discussion on the role of mechanisms in scientific explanations. For example, Craver (2007) bases his account of mechanisms on similar schema, where the functioning of higher-level mechanisms usually depends on the intrinsic causal properties of the lower-level parts. One of the benefits of reductionistic heuristics is that they increase the relative tractability and predictability of systems by making the previously mentioned assumptions of their structure and context. In general, these reductionistic heuristics tend to underestimate the importance of environment and take the system functionality to be mostly 
context-independent (Wimsatt 2007). As we will see in the next section, this assumption does not always hold for rationally designed systems.

The success of rational design depends on the availability of well-characterized, modular parts. Synthetic biologists' pursuit of modular parts and systems should not, however, be confounded with the descriptive question on whether natural biological systems are modular or not. As Knuuttila and Loettgers (2013b) note, the modularity of natural biological systems is not a prerequisite for synthetic biology, though it would be beneficial for synthetic biologists. Many standardized parts are modeled on functionally similar natural biological structures, such as gene promoters, inducers or repressors. However, natural biological systems do not lend themselves automatically to synthetic biology (Andrianantoandro et al. 2006). Therefore, synthetic biologists either need to heavily modify natural components or construct completely novel components for standardization. The aim of these modifications is to increase the insulation of the modules by removing unwanted interactions and by making functional modules structurally isolated (Guimaraes, Liu, and Arkin 2013). Insulation and well-defined structural boundaries should make modules easier to replace, remove and recompose. Consequently, Sune Holm (2015) argues that synthetic biologists take the mechanistic or machine-like nature of biological systems as a heuristic goal, but do not themselves consider natural biological systems to be similar to man-made artifacts.

This relates to one of the major methodological differences between engineering-dominated rational design and more biologically-inspired design methods, such as directed evolution: how does one approach the inherent complexity and variation of biological systems (see Giese et al. 2013)? Proponents of the rational design approach try to suppress variation by modifying their parts and systems to be more machine-like. In turn, as I will discuss in Section 3, evolutionary approaches take the variation, and the "emergent" properties that arise from the system's integrative structure, to be a fundamental part of living systems, and aim to utilize them in their design process. 


\subsection{The limitations of rational design}

The limitations of rational design become apparent in cases where the requirements of the partcentered rational design approach are not met, or in cases where reductionistic heuristics fail. I delineate three types of problem for rational design: lack of mechanistic detail (failure of understanding), context-sensitivity (failure of standardization), and change in functionality due to evolution (failure of stability).

As a parts-based approach, rational design requires extensive structural information and mechanistic details of its components. If the functionalities of the used components or the used model (e.g. the host bacteria strain) are not known, promiscuous functions might arise and affect the system behavior. This is not an outside possibility, as in all bacteria there are a great number of genes with unknown functions. For example, in a recent research on minimal genomes, J. Craig Venter's research group were able to create a Mycoplasma strain with a minimal genome of 473 genes (Hutchison et al. 2016). Even in their simplified model, they could not determine the function of $31 \%$ of the genes.

Synthetic biology can quite reliably define a limited toolbox of standardized parts, and use them to create very simple devices such as detectors, oscillators or reporter circuits. Unfortunately, the next step of combining well-defined simple devices to more complex multi-functional devices or even whole systems is exponentially more difficult (Purnick and Weiss 2009). Each standardized part has a limited domain range where it can be implemented predictably. Synthetic biologists are, therefore, limited by their ability to predict what effects the component has on system behavior and how this behavior changes in a new biological context (Blake and Isaacs 2004). Biological systems and environments are highly variable and are riddled with molecular noise, and susceptible to the emergence of nonlinear interactions. Knuuttila and Loettgers (2014) argue that the ubiquity of stochastic noise is one of the major differences between living and engineered systems. It is also one of the main obstacles for the engineering-dominated synthetic biology and rational design 
approach. In biological systems, even well-characterized components might show unpredictable behavior or fail to function when they are transferred to a new context. Cardinale and Arkin (2012) distinguish several potential context changes that might affect the functionality of components and systems of synthetic biology, including, change of spatial location, host organism, or extracellular factors, such as temperature or nutrients.

Rational design is, however, able to overcome some limitations of context-dependence. One solution is to describe and define the functional ranges of each standardized component with enough detail to at least mitigate the effects of some context-dependencies, such as environmental effects (Güttinger 2013). This should define the environments or hosts where these types of components are used, or the metabolic requirements of the system. However, all these ways to overcome context-dependence require higher analysis and better characterization of standardized components, which is not always possible and in some cases is costly. Therefore, an alternative solution is "kludging," tinkering with the system to find a particular solution that provides the desired functionality (O’Malley 2011). Though typical of practice in molecular biology and biotechnology, kludging and its ad hoc solutions, however, offer another example how current synthetic biology diverges from the ideals of engineering-dominated synthetic biology, and its attempts to separate design from fabrication. ${ }^{1}$

${ }^{1}$ Tinkering and kludges should not be seen, however, as a plain disanalogy between synthetic biology and engineering. Even though systematized and autonomous design can be seen as an engineering ideal for the engineering-dominated conception of synthetic biology, kludging and similar ad hoc solutions that prioritize functionality over understanding are common in actual engineering practice. 
Finally, synthetic devices are susceptible to evolutionary change, just like natural biological systems. As synthetic systems are primarily implemented in bacteria, they go through hundreds of generations which inevitably leads to mutational change. Natural evolution is destructive to synthetic systems, "relentlessly chipping away at painstakingly engineered networks and allowing the organism to escape control.” (Dougherty and Arnold 2009, 486). Engineered objects of synthetic biology are especially susceptible to evolutionary change. They are constructed to perform functions that are primarily beneficial to humans, not to organisms themselves. Therefore, synthetic organisms might have lower fitness than either wild-type organisms, or some mutant strain that develops from the synthetic organism. In general, evolution degrades the functionality of synthetic circuits and non-functional mutants have a tendency to take over a population of synthetic bacteria as natural selection favors mutants with less metabolic burden (Arkin and Fletcher 2006). Although in some cases these new functions might be beneficial, they are more often in contradiction with the design goals and are unwelcomed by researchers. In the most extreme cases, synthetic systems might evolve new functions that are actually harmful to humans or other organisms, and therefore, the ability of synthetic devices to go through evolution imposes questions to biosafety.

I have analyzed the limitations of the rational design approach. They are first and foremost the limitations of their parts, and the limitations of reductionistic approaches in general. However, living systems do not fulfill the requirements of design easily. The decomposition of organisms and their complex interconnected molecular interactions to salient functions, and mapping these functions to relatively simple modules and parts is already a difficult task. This problem is accentuated for synthetic biologists, who attempt to recompose these modular parts to apply to more complex systems (Knuuttila and Loettgers 2013b). Several biological components are far from the machine-like plug and play ideal, as they exhibit overlapping, unintended variation and contextdependence (Porcar and Peretó 2016). 
As such, rational design is currently best applied in cases where a collection of well-characterized components exists, the constructs are relatively simple, and the environment can be controlled. The application of rational design has been most fruitful in domains that exhibit hierarchical architecture with relatively simple modules and input-output relationships between their parts. One example of such domains is the design of genetic circuits and regulatory networks, where regulatory and constitutive genes and their promoters have been combined with simple modules with specified behavior, such as switches, cascades, circuits, and oscillators (see Elowitz and Leibler 2000; Purnick and Weiss 2009).

\section{Directed evolution}

Engineering-dominated synthetic biology is at odds with natural evolution. As I discussed previously, evolution ruins the good work of the designer by chipping away at the functionality of a system, leading to a loss of function or to promiscuous functionalities. The inherent tendency of natural systems to evolve away from engineered behavior hinders the realization of rational design goals. This unpredictability and stochasticity of evolving biological systems highlights one of the major disanalogies between them and engineered systems, as engineering presumes a level of control that is rarely achieved in living systems (Knuuttila and Loettgers 2014). This motivates the use of reductionistic heuristics and attempts to conceptualize systems as machine-like, thus making them more suitable for rational design (Holm 2015).

Furthermore, as far as natural evolution can be considered a designer itself, it is unpredictable and sub-optimal from the perspective of synthetic biology. Natural evolution finds solutions that are good enough for the purposes in the given environment, but not necessarily the most efficient ones. Improvement or optimization of natural biological organisms is actually seen as one of the aims of synthetic biology (see (Endy 2005)). However, the task of improving designs of natural selection does not entail rejecting some of the ideas of natural selection as a design principle. As discussed 
previously, rational design faces some limitations and cannot achieve the effective design of systems in all cases.

Rational design is, therefore, often complemented or replaced with other design approaches, which take their inspiration from natural evolution. The most widely used evolutionary design method is directed evolution, which aims to use random mutations and deliberate selection to guide the designed system to evolve in a desired direction (Blake and Isaacs 2004). Design through directed evolution proceeds by iterating between two stages: the library generation and the selection/screening stage (Marguet et al. 2007). In the first stage, random mutations are induced into a target sequence (e.g., through error-prone replication, PCR errors or DNA shuffling) to create a library of mutants that exhibit relevant variation. In the second stage, the generated library is screened, and one or more mutants, with desired phenotypic differences, are selected for further iterations. The library-generation and screening/selection cycle is repeated until a desired endpoint is achieved. As Lewens (2013) notes, this process resembles the artificial selection used in agriculture and animal husbandry.

Due to its similarity to natural evolution and its reliance on random mutations, directed evolution is sometimes taken to be inferior to rational design, especially in engineering-dominated conceptions of synthetic biology. Directed evolution sacrifices some mechanistic understanding and some tractability of the system to increase functionality. Therefore, some proponents of rational design frame directed evolution not as an autonomous design approach, but as a way to supplement rational design by providing kludges to non-functioning rationally designed systems. Bujara and Panke (2010), for example, consider directed evolution to be an intermediate step to overcome the current limitations of rational design. For engineering-dominated synthetic biology, directed evolution is a temporary kludge that allows synthetic biologists to design functional systems until the methods of rational design and its toolbox of standardized parts are sufficiently well developed. Subsequently, directed evolution is often considered a less rational form of design, and is described 
as an "irrational" (Lewens 2013, 642) or "semi-rational" (Guimaraes, Liu, and Arkin 2013, 72) approach, or even "engineering without design” (Marguet et al. 2007, 610).

Behind this juxtaposition between engineering-inspired rational design and biologically-inspired (irrational) directed evolution lies the previously discussed philosophical question of how synthetic biology should approach "living" features of biological systems. Whereas rational design tries to suppress variation and take natural evolution to be first and foremost a destructive force, directed evolution aims to utilize variation and evolution in its design process (Giese et al. 2013). Proponents of the engineering-dominated conception of synthetic biology consider that the application of engineering principles to biology will bring control and simplicity to inherently complex and non-modular biological systems (see (Andrianantoandro et al. 2006; Endy 2005)). In turn, a few critics have argued that this "mechanization" of living systems "would actually destroy the essential characteristics of living matter" (Schmidt 2015, 21) or at least downplay the importance of properties like self-organization and variation in biological systems (Oftedal and Parkkinen 2013). However, these appeals to the indispensable emergent properties of living systems have been accused of promoting vitalism, whereas synthetic biology must "shatter the notion that biological systems are fundamentally special in some way that prevents them from being rationally engineered" (Agapakis and Silver 2009, 711).

In this section, I will discuss the methods and application of directed evolution more thoroughly. As I proceed to argue, directed evolution is more than just an efficient way of tinkering. It is a systematic design approach that has its own benefits, limitations, and suitable domains of application in contrast to rational design.

\subsection{The requirements and benefits of directed evolution}

Directed evolution requires a different conceptualization of the design task in comparison to the rational design approach. Unlike rational design, directed evolution does not proceed from parts and 
their sub-functions to a system with a composite function. Rather, in directed evolution the designer's main tasks are the choice of the mutational target, the choice of appropriate screening and selective pressures, and to decide when the system has evolved to a desired endpoint. Directed evolution does not construct systems de novo, but requires some kind of a starting point where the manipulations and modifications are made. This idea of modifying existing structures rather than constructing them bottom-up shows why directed evolution is well suited to complement other design approaches. A commonly used starting point and mutational target is a non-functional system originally designed by rational design (O’Malley 2011). Therefore, directed evolution offers a more systematic method for tinkering with non-functional systems and finding the necessary kludges.

Methodologically, the application of directed evolution requires the ability to induce mutations, and to screen and select the produced phenotypes. Induced, mutations need to be detected and this requires mutations to be non-neutral, i.e. that they cause differences in phenotype, and that there are methods to detect these differences (Cambray, Mutalik, and Arkin 2011). Usually, this detection is done by selecting appropriate screening or selection methods. With screening, suitable organisms are analyzed and chosen for further iterations individually. The use of selection method, in turn, permits all organisms to be tested simultaneously, and those that do not exhibit the desired behavior are weeded out (Jäckel, Kast, and Hilvert 2008). Selection allows the synthetic biologist to discount a large set of unfruitful mutations, but it is also more experimentally demanding than just screening individual mutations. Both of these methods, however, require the designer to define the criteria for assessing success in each design cycle, and to determine when the desired endpoint is reached.

Besides the methodological requirements, directed evolution requires certain features from the target system. In the simplest form, an incremental pathway of beneficial mutations from the starting point to the desired behavior has to exist (Dougherty and Arnold 2009). As directed evolution proceeds through iteration of the library generation and selection/screening cycle, it 
requires detectable incremental changes in functionality. This can be helped by a proper definition of success criteria in the selection/screening process, possibly guided by mathematical modeling so that the designer can anticipate what kind of phenotypic changes are seen as beneficial for achieving the design goals (Blake and Isaacs 2004; Dougherty and Arnold 2009).

Directed evolution has certain benefits that overcome some of the limitations of rational design. First, directed evolution can be used in multiple levels of biological hierarchy (Marguet et al. 2007). It does not entail a bottom-up part-based approach to design, where characterization of parts is the first stage, and higher modules, devices and system can be constructed only through the fabrication and manipulation of parts. However, application of directed evolution becomes much harder when the target system is more complex. Currently the threshold of complexity is on the level of individual cells, and the design of multicellular systems is technically unfeasible (Porcar 2010). Second, directed evolution does not require as thorough an understanding of the system, the environment, and their interactions. It is, therefore, well suited to be applied in cases when there is incomplete knowledge of mechanistic details of the system, or when there are unpredictable interactions, possibly through emergent phenomena or due to the variability of the environment (Dougherty and Arnold 2009).

Finally, directed evolution can mitigate the effects of context-sensitivity by tuning the systems to a given environment. Directed evolution allows synthetic biologists to circumvent the effects of environmental variation by finding particular solutions for each environment. Multiple iterations of library generation and screening/selection cycles should allow synthetic biologists to carefully choose mutants that exhibit better functionality in the given environment. This ability to tune nonfunctional systems to achieve desired functionality in novel environments is one of the main rationales for the use of directed evolution (Haseltine and Arnold 2007). 
In addition to improving non-functional systems, environmental tuning via directed evolution has been proposed as a biosafety tool (Marliere 2009; Schmidt 2010). An example of this is the promotion of trophic containment and synthetic auxotrophs (Torres et al. 2016). In these experiments, bacteria are grown in a culture that includes molecules that do not occur in the natural food chain. These "alien nutrients" (for example, synthetic coenzymes) form a selective medium, and bacteria are forced to evolve so that their metabolism adapts to utilize the added nutrients. The goal of the process is to develop a mutant lineage that requires the exogenous supply of non-natural nutrients (Marliere 2009). This should prevent bacteria from growing in a natural environment which lacks the necessary nutrients, and, thus, decreasing the risk of accidental release of GM bacteria outside the laboratory. Environmental tuning using selective nutrients also highlights one of the potential trade-offs of directed evolution. Like tinkering and kludging, directed evolution prioritizes functionality over mechanical understanding and tractability.

\subsection{The limitations of directed evolution}

Directed evolution is most suited to cases where mutations cause relatively easily discernible changes in the phenotype. Qualitative changes, such as new metabolic properties, are easier to detect than quantitative changes. In the case of trophic containment, there is an unambiguous and effective selection setup that allows scientists to determine the success of the design cycle. Only mutants with desired metabolic functionality should be able to grow in the culture with added selective nutrients. However, the application of directed evolution becomes more difficult when the changes in the phenotype must be scrutinized in each iteration of the library-generation/selection cycle.

Analogous to the use of artificial selection in agriculture and animal husbandry, some cases and species are more suited for improvement through directed evolution. For example, the fitness landscape of the system affects the applicability of directed evolution. First, for directed evolution 
to reach a desired outcome, a pathway of incremental mutations to it from the starting sequence has to exist (Dougherty and Arnold 2009). In most cases, these mutations must also cause detectable changes in phenotype that allow synthetic biologists to pick the desired mutants for further iterations. This is further complicated by the fact that often screening for independent properties is difficult (Haseltine and Arnold 2007). Second, if the fitness landscape has many peaks, it is hard to discern whether the designed system is a local or a global maximum (Romero and Arnold 2009). The latter problem is dependent on the design task, and in many cases synthetic biologists only require the system to achieve good enough functionality, but not necessarily the best possible solution. However, the failure to reach an optimal solution is not limited only to directed evolution, and it is likely that rational design will likewise produce designs that are merely "good enough" and not the most efficient that could be achieved.

Due to these limitations and requirements on the fitness landscape of the system, one fruitful application of directed evolution has been in the domain of protein design. The rational design of novel proteins (such as enzymes) is a highly difficult and computationally demanding task, as it requires the designer to be able to model how genetic changes in protein coding regions affect the functional three-dimensional structure of the proteins. Directed evolution allows synthetic biologists to sample multiple protein variants and choose those with improved functionality without detailed knowledge of the functional consequences of genotypic change (Jäckel, Kast, and Hilvert 2008).

However, this reveals another challenge to directed evolution, which is the possible combinatorial complexity that arises from the design process. The number of possible mutational pathways increases exponentially through multiple cycles of iterations (Romero and Arnold 2009). This can increase the intractability of the design process and complicate the assessment of the successfulness of the design. If multiple mutations are needed to reach the peak of a fitness landscape, exploration of all solutions is not practically possible, although this can be alleviated through modeling (François and Hakim 2004; Romero and Arnold 2009). Furthermore, a properly developed selection 
setup helps decrease the combinatorial complexity that arises as the byproduct of the directed evolution design cycle. It allows the synthetic biologist to discount sets of unfruitful mutants in each iteration of selection.

Finally, design by directed evolution can lead to the increase in the context-sensitivity and the decrease of the generality of the system. As discussed in the previous section, directed evolution is used to mitigate the effects of context-sensitivity by environmental tuning (Haseltine and Arnold 2007). Directed evolution does not decrease the context-sensitivity of its systems, but rather, it allows the synthetic biologist to circumvent those effects by forcing the system to adapt to that specific environment. Evolutionary methods, such as the use of genetic algorithms, lend some problem solving to the environment and lead to closer coupling between system and its environment (Kuorikoski and Pöyhönen 2013). This environmental tuning, however, can make a system more context-sensitive and less likely to work properly in other environments, therefore decreasing the generality of designed systems to achieve functionality.

Sometimes the increased context-sensitivity of the system can be beneficial. For example, in the case of trophic containment, the increased context-sensitivity is itself the design goal (Marliere 2009). However, in most cases, context-sensitivity complicates the design work and restricts the implementation and reuse of synthetic components and systems. Furthermore, evolutionary methods do not merely increase the coupling between the system and the environment. They might also increase the coupling between parts of a system, thus increasing its integration and nonmodularity. As discussed in Section [sec:21], this makes targeted interventions more difficult and decreases the synthetic biologist's ability to manipulate and control the target, as well as rearrange or replace its parts. As small changes can propagate through a non-modular system unpredictably, this also decreases their tractability and scientists' ability to gain mechanical understanding about them (Oftedal and Parkkinen 2013). How much evolutionary methods actually cause nonmodularity is, however, an empirical question, and differs from case to case. 


\section{Conclusions}

Synthetic biology is considered an amalgamation of previous biotechnology and engineering, as it aims to bring the methods and heuristics of the engineering sciences to help scientists construct and modify biological systems. Central to this is the design of new biological parts and systems through rational design. Rational design is a parts-based approach that proceeds to make biological systems more machine-like through decoupling design tasks and standardizing components. It is currently the most prominent design approach in synthetic biology. Consequently, proponents of the engineering-dominated conception of synthetic biology argue that well-developed synthetic biology should aim to make rational design its default design methodology.

This paper offers a contrary view, opposing the idea of rational design as a one-size-fits-all solution for design in synthetic biology. Neither should this "methodological monism" be seen as the goal of synthetic biology. Other design approaches, such as directed evolution, are widely used in actual research projects in synthetic biology. Rational design and directed evolution have important differences in their design methodology. I have assessed the respective benefits and limitations of rational design and directed evolution by providing a systematic analysis of the heuristics and methodological principles of each approach. Table [tab:1] describes the relative differences of both rational design and directed evolution design approaches. Rather than arguing for the primacy of either approach, this analysis shows that each approach has its own domain where it is best applied.

Rational design is best suited for cases where reductionistic heuristics, based on the functional decomposition of the system, are the most successful, that is, in cases where the behavior of the system can be described and modeled through the functionalities of its constituent parts. As such, the application of rational design attempts to suppress biological variation and emergent features of living systems, as well as the influence of the environment. In turn, directed evolution takes inherent complexity, noisiness, and biological phenomena to be an indispensable part of living 
systems (Giese et al. 2013). Evolution and biological variation are used as constructive forces in the design process. Directed evolution has been most successful in cases where the system is primarily required to achieve a certain functionality or efficiency, and possible differences in the system's performance and functionality are possible to discern, and subsequently, to screen and select.

This paper defends a pluralistic view on design in synthetic biology. I have suggested that, rather than seeing directed evolution as a supplement to, or as an impure form of, rational design, it should be seen as an alternative design approach that can complement rational design methods. Furthermore, one should recognize the diversity of design practices in synthetic biology, and therefore be cautious about defining synthetic biology merely as a project that aims to apply engineering principles to biology. Too high a focus on possible engineering ideals can be detrimental, and analyzing synthetic biology through its engineering-dominated conception is, in part, misleading. The diversity of complementary design approaches should not be seen as a limitation for synthetic biology, nor should biologically-inspired methods be disregarded as divergences from the engineering ideals. Rather, the integration of evolution and the utilization of complex features of living systems, such as self-organization and biological variation, can bring benefits and allow more powerful methods to design and construct biological devices for human purposes.

\section{References}

Agapakis CM (2014) Designing synthetic biology. ACS Synth Biol 3(3):121-128. https://doi. org/10.1021/sb4001068

Agapakis CM, Silver PA (2009) Synthetic biology: exploring and exploiting genetic modularity through the design of novel biological networks. Mol Biosyst 5(7):704-713.

https://doi.org/10.1039/B9014 84E

Andrianantoandro E, Basu S, Karig DK, Weiss R (2006) Synthetic biology: new engineering rules for an emerging discipline. Mol Syst Biol 2:1-14. https://doi.org/10.1038/msb4100073

Arkin AP, Fletcher DA (2006) Fast, cheap and somewhat in control. Genome Biol 7(8):114. https://doi. org/10.1186/gb-2006-7-8-114 
Bechtel W, Richardson RC (1993) Discovering complexity: decomposition and localization as strategies in scientific research. Princeton University Press, Princeton

Blake WJ, Isaacs FJ (2004) Synthetic biology evolves. Trends Biotechnol 22(7):321-324. https://doi. org/10.1016/j.tibtech.2004.04.008

Boudry M, Pigliucci M (2013) The mismeasure of machine: synthetic biology and the trouble with engi- neering metaphors. Stud Hist Philos Biol Biomed Sci 44(4):660-668

Bujara M, Panke S (2010) Engineering in complex systems. Curr Opin Biotechnol 21(5):586591. https ://doi.org/10.1016/j.copbio.2010.07.007

Cambray G, Mutalik VK, Arkin AP (2011) Toward rational design of bacterial genomes. Curr Opin Microbiol 14(5):624-630. https://doi.org/10.1016/j.mib.2011.08.001

Cameron DE, Bashor CJ, Collins JJ (2014) A brief history of synthetic biology. Nat Rev Microbiol 12(5):381-390. https://doi.org/10.1038/nrmicro3239

Cardinale S, Arkin AP (2012) Contextualizing context for synthetic biology-identifying causes of fail- ure of synthetic biological systems. Biotechnol J 7(7):856-866.

https://doi.org/10.1002/biot.20120 0085

Craver CF (2007) Explaining the brain: mechanisms and the mosaic unity of neuroscience. Oxford Uni- versity Press, Oxford

Dougherty MJ, Arnold FH (2009) Directed evolution: new parts and optimized function. Curr Opin Bio- technol 20(4):486-491. https://doi.org/10.1016/j.copbio.2009.08.005

Elowitz MB, Leibler S (2000) A synthetic oscillatory network of transcriptional regulators. Nature 403(6767):335-338. https://doi.org/10.1038/35002125

Endy D (2005) Foundations for engineering biology. Nature 438(7067):449-453. https://doi.org/10.1038/ nature04342

ERASynBio (2014) Next steps for European synthetic biology: a strategic vision from ERASynBio. Technical report, ERASynBio/European Commission

François P, Hakim V (2004) Design of genetic networks with specified functions by evolution in silico. PNAS 101(2):580-585. https://doi.org/10.1073/pnas.0304532101

Giese B, Koenigstein S, Wigger H, Schmidt JC, von Gleich A (2013) Rational engineering principles in synthetic biology: a framework for quantitative analysis and an initial assessment. Biol Theory 8(4):324-333. https://doi.org/10.1007/s13752-013-0130-2

Guimaraes JC, Liu CC, Arkin AP (2013) From biological parts to circuit design. In: Zhao H (ed) Syn- thetic biology: tools and applications. Elsevier, Amsterdam, pp 63-78

Güttinger S (2013) Creating parts that allow for rational design: synthetic biology and the problem of context-sensitivity. Stud Hist Philos Biol Biomed Sci 44(2):199-207. https://doi.org/10.1016/j.shpsc .2013.03.015

Haseltine EL, Arnold FH (2007) Synthetic gene circuits: design with directed evolution. Annu Rev Bio- phys Biomed 36:1-19. https://doi.org/10.1146/annurev.biophys.36.040306.132600 
Heinemann M, Panke S (2006) Synthetic biology-putting engineering into biology. Bioinformatics 22(22):2790-2799. https://doi.org/10.1093/bioinformatics/btl469

Heinemann M, Panke S (2009) Synthetic biology: putting engineering into bioengineering. In: Fu P, Panke S (eds) Systems biology and synthetic biology. Wiley, New York, pp 387-409

Holm S (2015) Is synthetic biology mechanical biology? Hist Philos Life Sci 37(4):413-429. https://doi. org/10.1007/s40656-015-0081-y

Houkes W, Vermaas PE (2010) Technical functions: on the use and design of artefacts. Springer, Dordrecht

Hutchison CA, Chuang RY, Noskov VN, Assad-Garcia N, Deerinck TJ, Ellisman MH, Gill J, Kannan K, Karas BJ, Ma L, Pelletier JF, Qi ZQ, Richter RA, Strychalski EA, Sun L, Suzuki Y, Tsvetanova B, Wise KS, Smith HO, Glass JI, Merryman C, Gibson DG, Venter JC (2016) Design and synthesis of a minimal bacterial genome. Science 351(6253):aad6280.

https://doi.org/10.1126/science.aad6253

Jäckel C, Kast P, Hilvert D (2008) Protein design by directed evolution. Annu Rev Biophys 37:153-173. https://doi.org/10.1146/annurev.biophys.37.032807.125832

Knuuttila T, Loettgers A (2013a) Basic science through engineering? synthetic modeling and the idea of biology-inspired engineering. Stud Hist Philos Biol Biomed Sci 44(2):158-169. https://doi. org/10.1016/j.shpsc.2013.03.011

Knuuttila T, Loettgers A (2013b) Synthetic modeling and mechanistic account: material recombination and beyond. Philos Sci 80(5):874-885. https://doi.org/10.1086/673965

Knuuttila T, Loettgers A (2014) Varieties of noise: analogical reasoning in synthetic biology. Stud Hist Philos Sci 48:76-88. https://doi.org/10.1016/j.shpsa.2014.05.006

Kroes P (2012) Technical artefacts: creations of mind and matter-a philosophy of engineering design. Springer, Heidelberg

Kuorikoski J, Pöyhönen S (2013) Understanding nonmodular functionality: lessons from genetic algo- rithms. Philos Sci 80(5):637-649. https://doi.org/10.1086/673866

Lewens T (2013) From bricolage to biobricksTM: synthetic biology and rational design. Stud Hist Philos Biol Biomed Sci 44(4):641-648. https://doi.org/10.1016/j.shpsc.2013.05.011

Marguet P, Balagadde F, Tan C, You L (2007) Biology by design: reduction and synthesis of cellular components and behaviour. J R Soc Interface 4(15):607-623.

https://doi.org/10.1098/rsif.2006.0206

Marliere P (2009) The farther, the safer: a manifesto for securely navigating synthetic species away from the old living world. Syst Synth Biol 3(1-4):77. https://doi.org/10.1007/s11693009-9040-9

Oftedal G, Parkkinen VP (2013) Synthetic biology and genetic causation. Stud Hist Philos Biol Biomed Sci 44(2):208-216. https://doi.org/10.1016/j.shpsc.2013.03.016

O’Malley M (2011) Exploration, iterativity and kludging in synthetic biology. Comptes Rendus Chim 14(4):406-412. https://doi.org/10.1016/j.crci.2010.06.021 
O'Malley M, Powell A, Davies JF, Calvert J (2007) Knowledge-making distinctions in synthetic biology. Bioessays 30(1):57-65. https://doi.org/10.1002/bies.20664

Packer MS, Liu DR (2015) Methods for the directed evolution of proteins. Nat Rev Genet 16(7):379-394. https://doi.org/10.1038/nrg3927

Porcar M (2010) Beyond directed evolution: darwinian selection as a tool for synthetic biology. Syst Synth Biol 4(1):1-6. https://doi.org/10.1007/s11693-009-9045-4

Porcar M, Peretó J (2016) Nature versus design: synthetic biology or how to build a biological non- machine. Integr Biol 8(4):451-455. https://doi.org/10.1039/C5IB00239G

Purnick PEM, Weiss R (2009) The second wave of synthetic biology: from modules to systems. Nat Rev Mol Cell Biol 10(6):410-422. https://doi.org/10.1038/nrm2698

Romero PA, Arnold FH (2009) Exploring protein fitness landscapes by directed evolution. Nat Rev Mol Cell Biol 10(12):866-876. https://doi.org/10.1038/nrm2805

Roosth S (2017) Synthetic: how life got made. University of Chicago Press, Chicago

Schmidt M (2010) Xenobiology: a new form of life as the ultimate biosafety tool. Bioessays 32(4):322- 331. https://doi.org/10.1002/bies.200900147

Schmidt JC (2015) Synthetic biology as late-modern technology. In: Giese B, Pade C, Wigger H, von Gleich A (eds) Synthetic biology, risk engineering. Springer, Heidelberg, pp 1-30

Simon HA (1996) The sciences of the artificial, 3rd edn. MIT Press, Cambridge

Torres L, Krüger A, Csibra E, Gianni E, Pinheiro VB (2016) Synthetic biology approaches to biological containment: pre-emptively tackling potential risks. Essays Biochem 60(4):393410. https://doi.org/10.1042/EBC20160013

Wagner A (2005) Robustness and evolvability in living systems. Princeton University Press, Princeton

Wimsatt WC (2007) Re-engineering philosophy for limited beings: piecewise approximations to reality. Harvard University Press, Cambridge 